\title{
MicroRNA-21 regulates the biological behavior of esophageal squamous cell carcinoma by targeting RASA1
}

\author{
XIAOHUA CHEN $^{1 *}$, SINA CAI $^{2 *}$, BAOXIA LI $^{3 *}$, XIAONA ZHANG $^{4}$, WENHUI LI $^{1}$, \\ HENGLUN LIANG $^{1}$, XIAOLONG CAO ${ }^{1}$, LIPING WANG ${ }^{5}$ and ZIQING WU ${ }^{6}$ \\ ${ }^{1}$ Department of Oncology, Panyu Central Hospital, Cancer Institute of Panyu, Guangzhou, Guangdong 511400; \\ ${ }^{2}$ Department of Oncology, The Third Affiliated Hospital of Southern Medical University, Guangzhou, Guangdong 510630; \\ ${ }^{3}$ State Laboratory of Oncology in South China, Sun Yat-Sen University Cancer Center, Guangzhou, Guangdong 510060; \\ ${ }^{4}$ Graceland Medical Center, The Sixth Affiliated Hospital of Sun Yat-Sen University, Guangzhou, Guangdong 510655; \\ ${ }^{5}$ Department of Clinical Oncology, The First City Hospital of Chenzhou, Chenzhou, Hunan 423099; \\ ${ }^{6}$ Department of Pathology, Integrated Hospital of Traditional Chinese Medicine, Southern Medical University, \\ Guangzhou, Guangdong 510310, P.R. China
}

Received June 20, 2018; Accepted December 5, 2018

DOI: $10.3892 / o r .2018 .6944$

\begin{abstract}
MicroRNA-21 (miR-21) has been revealed to play a crucial role in regulating the biological behavior, including proliferation, migration, invasion and metastasis in certain cancers. However, its role in esophageal squamous cell carcinoma (ESCC) has yet to be elucidated. Based on the data of GSE13937 downloaded from Gene Expression Omnibus (GEO) database, miR-21 was revealed to be one of the top 20 differentially expressed (DE) miRNAs screened using the Morpheus online tool. RAS p21 protein activator 1 (RASA1) was predicted as the target gene of miR-21 using the predicting software and was combined with miR-21 using the luciferase reporter assay. Its relative expression was significantly decreased, however, miR-21 was increased in the tumor tissues compared to the normal adjacent tissues in patients with ESCC as determined by quantitative polymerase chain reaction (q-PCR). Furthermore, overexpression of miR-21 (mimic) could significantly decrease the gene level of RASA1. Conversely, downregulation of miR-21 (inhibitor) significantly
\end{abstract}

Correspondence to: Dr Xiaohua Chen, Department of Oncology, Panyu Central Hospital, Cancer Institute of Panyu, 8 Fuyu Road, Qiaonan Street, Guangzhou, Guangdong 511400, P.R. China E-mail: cxh0663@126.com

Dr Ziqing $\mathrm{Wu}$, Department of Pathology, Integrated Hospital of Traditional Chinese Medicine, Southern Medical University, 13 Courtyard, Shiliugang Road, Haizhu, Guangzhou, Guangdong 510310, P.R. China

E-mail: hailian@fimmu.com

*Contributed equally

Key words: esophageal squamous cell carcinoma, microRNA, proliferation, invasion, migration increased the gene level of RASA1, while downregulation of RASA1 (siRASA1) markedly increased the gene expression of miR-21. Notably, the expression of Snail and vimentin were significantly increased by upregulation of miR-21 and downregulation of RASA1. Transwell results revealed that miR-21 and RASA1 regulated proliferation, migration and invasion in ESCC cells. In an in vivo model, miR-21 inhibitor (antagomir) could inhibit tumor growth. In conclusion, miR-21 regulated cell proliferation, migration, invasion and tumor growth of ESCC by directly targeting RASA1, which may have been achieved via regulation of Snail and vimentin. Anti-miR-21 revealed an antitumor effect. Thus, it may be considered as a possible target for ESCC therapy.

\section{Introduction}

Esophageal carcinoma (EC) constitutes one of the most common types of cancer with high mortality and incidence worldwide (1). It is ranked fourth in mortality in China. Esophageal squamous cell carcinoma (ESCC) is the predominant histological type and represents approximately $90 \%$ of all cases in China (2). ESCC patients always have a low survival rate since they are diagnosed at an advanced stage or even with distant metastasis $(3,4)$. Metastasis has become the main cause of death in these patients. Therefore, elucidating the molecular mechanism concerning its biological behaviour, such as metastasis, is essential for EC treatment.

MicroRNAs (miRNAs) are approximately 20-22 nucleotides molecules and have been reported to play an important role in tumorigenesis and development in certain cancers including ESCC (5-9). MircroRNA-21 (miR-21) has been revealed to be highly expressed in many solid tumors such as ESCC, oral squamous cell carcinoma (OSCC), and breast, lung and gastric cancer (10-17). It may therefore be considered as a new biomarker for cancer diagnosis and a new target for cancer treatment. A recent study revealed that serum miR-21 was related to the progression of ESCC (12). Studies on miR-21 
revealed that it regulated the invasion, migration and metastasis of cells (such as A549 and PC-3) $(9,18)$ and epithelial-mesenchymal transition (EMT), which is considered as the first step to metastasis (19-27). The biological behaviors of cells such as proliferation, migration and invasion have been regarded as precursors to metastasis. A previous study revealed that the expression of miR-21 was significantly altered in patients with ESCC as determined by microarray (28). miRNAs have been demonstrated to play a regulatory role by interacting with their target genes. The results from accurate prediction software (TargetScan, miRwalk, miRanda and PITA) revealed that RAS p21 protein activator 1 (RASA1) may be the target gene of miR-21. However, the role of miR-21 and its target gene in regulating the biological behavior of ESCC has yet to be elucidated.

In the present study, cell colonies assay, wound healing and Transwell assay were used to detect the role of miR-21 and its target gene in cell proliferation, migration and invasion of ESCC. We examined the interaction between miR-21 and RASA1 using a luciferase reporter assay. The relative gene expression levels of RASA1 and miR-21 were detected by quantitative polymerase chain reaction (q-PCR). Finally, the effect of miR-21 on ESCC growth in vivo was examined in a nude mouse model. Generally, in the present study, we aimed to identify the role of miR-21 in the regulation of ESCC cells and to reveal some new targets for cancer treatment.

\section{Materials and methods}

Microarray data. Gene expression profiles of GSE13937 were downloaded from the GEO repository. These data were based on the GPL8835 platform. The data of 44 patients with ESCC (including 44 samples of normal adjacent esophageal tissues and 44 samples of tumor tissues) were chosen to perform further analysis and create the heat maps with Morpheus online tool (https://software.broadinstitute.org/morpheus/). Then, the top 20 of differentially expressed (DE) miRNAs were screened. This study was published by Mathé et al (28). Total RNA was extracted and detected by miRNA microarray chips. Version 3. R (BioConductor) (http://www.bioconductor. org/install/) was used for background correction and normalization of the data.

Sample collection. All samples were collected at the Panyu Central Hospital and the Third Affiliated Hospital of Southern Medical University from February 27, 2010 to May 2, 2017. One patient was female and 19 patients were male, with a mean age of $62.4 \pm 5.8$ years, and diagnosed with clinicopathological characteristics of ESCC. These samples were used only for the detection of the gene expression of miRNA and 10 patient samples among the 20 were utilized for target gene detection. All samples were stored at $-80^{\circ} \mathrm{C}$ after collection.

Ethics statement. This study was approved by the Ethics Committee of Panyu Central Hospital (Guangzhou, China). All patients agreed to participate provided informed consent for this study.

Cell culture and reagents. The cell line Eca-109 (human esophageal squamous carcinoma cell) with metastatic ability and 293T cell were obtained from the State Laboratory of Oncology in South China, Sun Yat-Sen University Cancer Center (Guangzhou, China). The cell line KYSE510 (well differentiated squamous cell carcinoma cell line) was obtained from the Central Laboratory, Nanfang Hospital, Southern Medical University (Guangzhou, China). The Eca-109 and KYSE510 cells were cultured in RPMI-1640 media, supplemented with $10 \%$ fetal calf serum (FCS; Gibco; Thermo Fisher Scientific, Inc., Waltham, MA, USA) and were maintained under $5 \% \mathrm{CO}_{2}$ at $37^{\circ} \mathrm{C}$. The $293 \mathrm{~T}$ cells were cultured in Dulbecco's modified Eagle's medium (DMEM; Gibco; Thermo Fisher Scientific, Inc.), supplemented with 10\% FCS and 1\% GlutaMAX and 1\% penicillin with streptomycin.

Animals. Ten BALB/C nude mice aged 4-6 weeks old with a male to female ratio of 1:1 weighing 20-25 g were used in the present study. These mice were obtained from Chase Reward Ltd. (Guangzhou, China) and kept under the specific pathogen-free conditions (temperature, $20-26^{\circ} \mathrm{C}$; 12-h light-dark cycle; aseptic food and water). The animal experiments were approved by the Ethics Committee of Panyu Central Hospital (no. K20170002).

Cell transfection. miR-21 mimics (overexpression), miR-21 inhibitor (downregulation) and negative control (NC including mimic NC, inhibitor NC) were purchased from Guangzhou RiboBio Co., Ltd. (Guangzhou, China). Transfections were performed on Eca-109 cells with Lipofectamine 2000 (Invitrogen; Thermo Fisher Scientific, Inc.) following the manufacturer's protocol. Briefly, $5 \times 10^{5}$ cells were cultured in 6-well plates for $24 \mathrm{~h}$ prior to transfection. The miR-21 inhibitor, mimics and their NC nucleotides were added to the cell with transfection reagent to a final concentration of 50 and $100 \mathrm{~nm} / 1$, respectively. The cells were treated for $48 \mathrm{~h}$ and harvested for analysis.

Design and construction of eukaryotic expression vector for RASA1. miRWalk (miR Walk1.0) (http://zmf.umm. uniheidelberg.de/apps/zmf/mirwalk/micrornapredictedtarget. html) was utilized to predict the target gene and RASA1 was predicted as the target gene of miR-21. Thus, the vector for RASA1 was constructed. The 3' untranslated regions (3'UTR) of RASA1 mRNA were amplified using the PCR instrument (GeneAmp PCR System 2400; Applied Biosystems; Thermo Fisher Scientific, Inc.), which was bound to the mature miR-21 (MIMAT0000076). To prevent formation of a termination signal, 'taagcta' was selected as the region in a mutational expression vector template and was amplified (amplification primers are presented in Table I). Then, the aforementioned template was transfected with psi-CHECK-2 luciferase plasmid and used for dual-luciferase reporter assay.

Dual-luciferase reporter assay. The 3'UTR fragments of the RASA1 gene were amplified and then transfected and cloned into the psi-CHECK-2 luciferase miRNA expression reporter vector. The $293 \mathrm{~T}$ cells $\left(2 \times 10^{4}\right)$ were cultured in 24 -well plates and transfected with $50 \mathrm{nM}$ miR-21 mimics, $100 \mathrm{nM}$ inhibitor or NC, $0.5 \mu \mathrm{g}$ of psi-CHECK-2 luciferase reporter vector that contained the wild-type or mutant 3'UTR of RASA1 or empty plasmid. Transfections were performed using 
Table I. The primer sequence of the genes.

Gene

Primer $\left(5^{\prime}-3^{\prime}\right)$

\begin{tabular}{ll}
\hline RASA13' & UTR-F: ccgctcgagCAGCCTTCGCCCCAGTGTTCTG \\
RASA13' & UTR-R: ataagaatgcggccgcTAATCAATTATGCAAGATATCCC \\
mutation RASA1: & \\
RASA13' & UTR-mF: GTGAATAACTATGCCAGCAACCTTGATTCGATTCTGTGCAGGATATTTGCACTATTT \\
RASA13' & UTR-mR: ataagaatgcggccgcTAATCAATTATGCAAGATATCCC \\
RASA1-F: & GAACTTGGGAATGTACCTGAAC \\
RASA1-R: & TGTGCACCACGCTCATTAC \\
miR-21-F: & ACACTCCAGCTGGGTAGCTTATCAGACTGATG \\
miR-21-RT: & CTCAACTGGTGTCGTGGAGTCGGCAATTCAGTTGAGTCAACATC \\
miRNA-R: & CTCAACTGGTGTCGTGGA \\
U6-F: & CTCGCTTCGGCAGCACA \\
U6-R: & AACGCTTCACGAATTTGCGT \\
K-ras-F: & GAGGCCTGCTGAAAATGAC \\
K-ras-R: & GCTGTGTCGAGAATATCCAA \\
AKT-F: & ATCGCTTCTTTGCCGGTATC \\
AKT-R: & CTTGGTCAGGTGGTGTGATG \\
PI3K-F: & AGGGAGCGAGTGCCTTTTAT \\
PI3K-R: & AAGCCCTGCAGTCAACATCA \\
E-caherin-F: & GTACTCAAAGCCCAGAATCC \\
E-caherin-R: & CCCTCAACTAACCCCCTTTA \\
Vimentin-F: & CGCCAGATGCGTGAAATGG \\
Vimentin-R: & ACCAGAGGGAGTGAATCCAGA \\
Snail-F: & TGGTTGCTTCAAGGACACAT \\
Snail-R: & GTTGCAGTGAGGGCAAGAA \\
18s rRNA-F: & CCTGGATACCGCAGCTAGGA \\
18s rRNA-R: & GCGGCGCAATACGAATGCCCC \\
$\beta$-actin-F: & ACTCTTCCAGCCTTCCTTCC \\
$\beta$-actin-R: & GTACTTGCGCTCAGGAGGAG \\
\hline
\end{tabular}

F, forward; R, reverse; $\mathrm{mF}$, forward primer of the mutant RASA1; $\mathrm{mR}$, reverse primer of mutant RASA1; RT, reverse transcription primer of miR-21.

Lipofectamine 2000 (Invitrogen; Thermo Fisher Scientific, Inc.) according to the manufacturer's instructions. Forty-eight hours after transfection, the luciferase activity was assessed by the Dual-Luciferase Reporter Assay System (GloMax; Promega Corporation, Madison, WI, USA). The experiments were repeated in 3 independent experiments in duplicate.

Cell colonies assay. Eca-109 cells (100) were cultured in 6-well plates until colonies could be observed. Then the cells were fixed by $4 \%$ of triformol and stained with Giemsa for $15 \mathrm{~min}$ at room temperature (Nanjing Jiancheng Taihao Biotechnology Co.,Ltd., Nanjing, China). Finally, the clones were counted under a light microscope (magnification, x100). The clone which contained $>10$ cells was regarded as one colony formation, and the colony rate was calculated using the following formula: colony formation rate $=($ colony formation numbers/100) $\mathrm{x} 100 \%$.

Scratch assay. Eca-109 cells $\left(8.0 \times 10^{5}\right)$ were cultured in 6-well plates for $24 \mathrm{~h}$ and confluence $(>90 \%)$ without any vacant space. Subsequently the cells were scratched using 10- $\mu$ l tips with a wound midline of the culture well, and then replaced in $1 \%$ FBS DMEM. Finally, the difference in width of the wounds was measured at 0,24 and $48 \mathrm{~h}$, respectively to evaluate the migration of cells and the healing rate was calculated.

Cell migration and invasion assay. Cell migration activities were detected using Transwell migration. After the transfected Eca-109 or KYSE510 cells were cultured for $24 \mathrm{~h}$, the cells $\left(1 \times 10^{5}\right)$ were resuspended in serum-free media and cultured in the inserts $(8-\mu \mathrm{m}$ diameter pore size; Corning Inc., Corning, NY, USA), which were placed in the 6-wells plates with $10 \%$ FBS serum media. The cells migrated from the upper surface to the lower surface of the membrane after 48-h incubation. Subsequently, the migrated cells were fixed with $100 \%$ methanol and stained with $1 \%$ toluidine. Finally, these stained cells were counted in 5 random optical fields under a light microscope (magnification, x200). The cell invasion assay was similar to the cell migration assay except the Transwell membrane was pre-coated with $24 \mathrm{mg} / \mathrm{ml}$ Matrigel (Corning Inc.). 


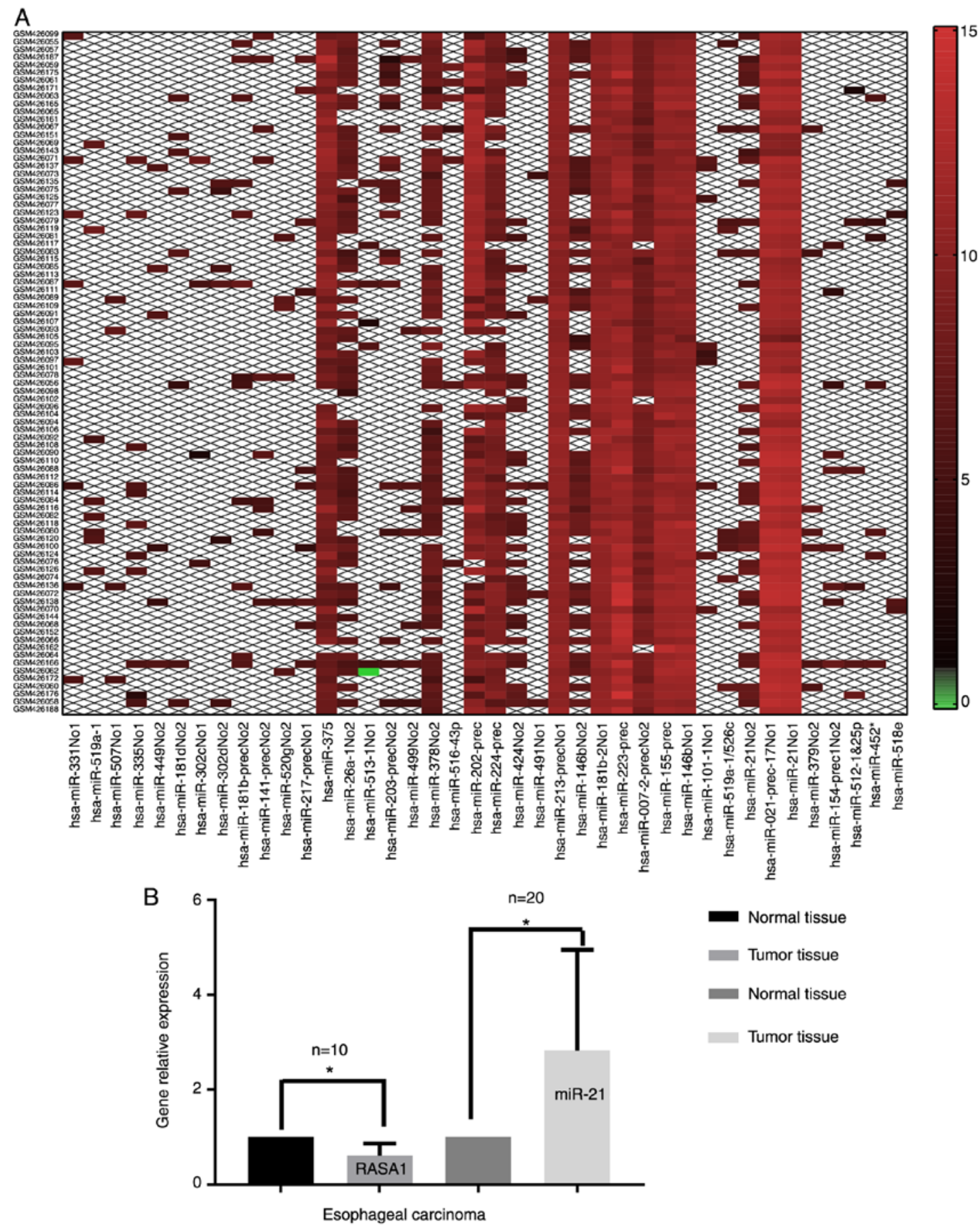

Figure 1. Heat map of the top 20 DE miRNAs screened using Morpheus online tool and the relative expression in tissues. (A) The results revealed that miR-21 was one of the top 20 DE miRNAs which was upregulated in patients with ESCC. The GSM identified with No. 1 represent the adjacent esophageal tissue, and No. 2 represent the tumor tissue. (B) The relative expression of miR-21 and RASA1 in the tumor tissues compared to the normal adjacent tissues in the patients with ESCC. A total of twenty patients with ESCC were enrolled in this study. The results revealed that the expression of miR-21 was significantly increased in the tumor tissues while RASA1 was significantly downregulated ( $\mathrm{P}<0.05)$. DE, differentially expressed; miR-21, microRNA-21; ESCC, esophageal squamous cell carcinoma.

Quantitative real-time PCR. Transfected Eca-109 cells and tissues were processed in TRIzol reagent (Invitrogen; Thermo Fisher Scientific, Inc.) and total RNA was extracted according to the manufacturer's instructions. First-strand cDNA was synthesized with $1 \mu \mathrm{g}$ total RNA per sample using the PrimeScript $^{\mathrm{TM}}$ RT Synthesis system (Takara Bio, Inc., Shiga, Japan). Subsequently, the cDNA sample was amplified using QuantiTect reagents (GeneCopoeia, Inc., Rockville, MD, USA) in a final volume of $20 \mu \mathrm{l}$ under the LightCycler 480II detector (Roche Diagnostics, Basel, Switzerland). The amplifications were performed as follows: predestination for $2 \min$ at $50^{\circ} \mathrm{C}$, denaturation for $30 \mathrm{sec}$ at $95^{\circ} \mathrm{C}$, followed by 40 cycles of $95^{\circ} \mathrm{C}$ for $5 \mathrm{sec}$, and $60^{\circ} \mathrm{C}$ for $34 \mathrm{sec}$. The melting curve analysis was performed to detect the specificity of amplification products. The experiments were performed in triplicate. U6 and $18 \mathrm{~S}$ rRNA genes were used as the miRNA-21 and RASA1 endogenous reference controls, respectively. $\beta$-actin was used for the rest of the genes as an endogenous reference control. The relative gene expression level was calculated using the $2^{-\Delta \Delta \mathrm{Cq}}$ method (29). Specific sense primers are presented in Table I. 

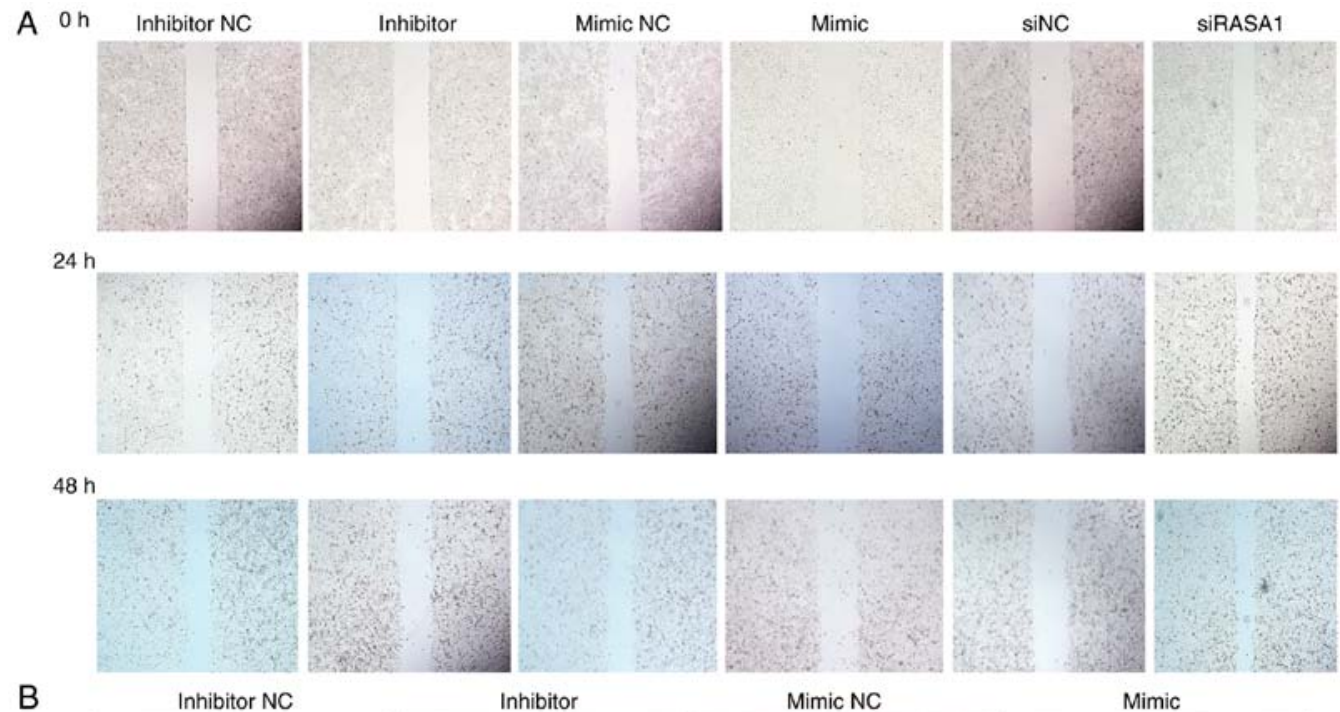

B
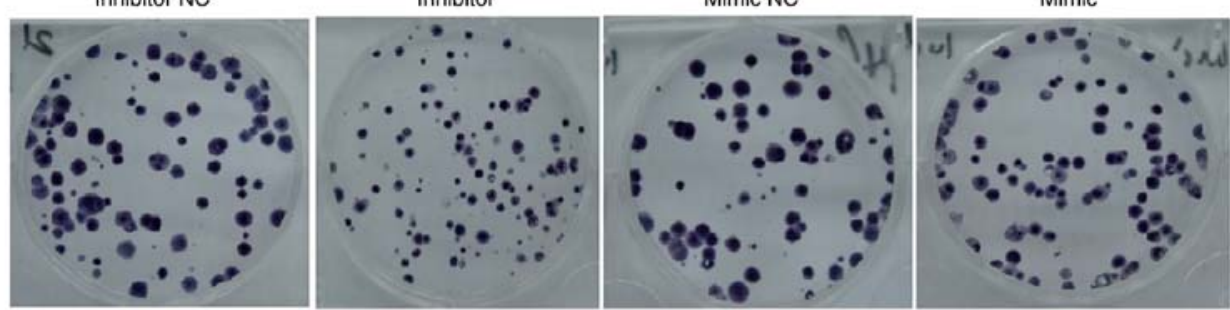

C
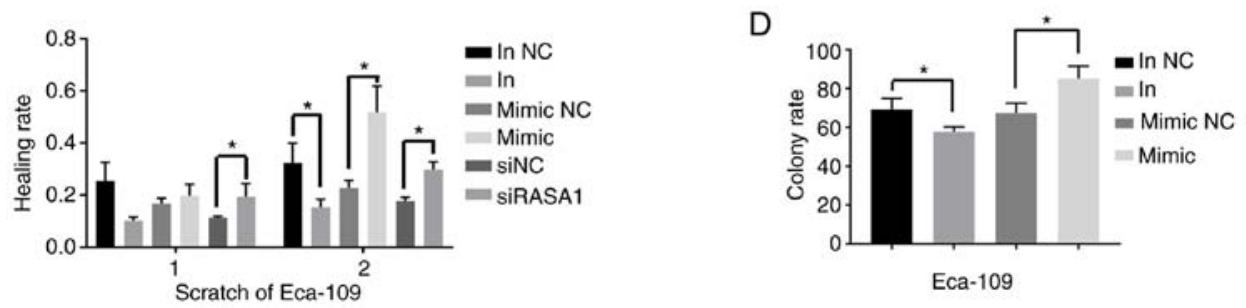

Figure 2. Effect of miR-21 on the colony formation rate and healing rate of ESCC in vitro. (A) Cells from a wound healing assay viewed under a microscope (magnification, x100). (B) Cells from a colony formation assay viewed under a microscope. (C) The cell healing rate of the miR-21 inhibitor group was significantly inhibited compared with the inhibitor NC group after the cells were transfected for $48 \mathrm{~h}$, while the healing rate was significantly enhanced in the miR-21 mimic and siRASA1 group compared with the mimic NC and the siNC group, respectively. 1 represents for $24 \mathrm{~h}, 2$ represents for $48 \mathrm{~h}$. (D) The cell colony formation rate was significantly decreased in the miR-21 inhibitor group compared with the inhibitor NC group, while the rate was significantly increased in the miR-21 mimic group compared with the mimic NC group ( $\mathrm{P}<0.05)$. miR-21, microRNA-21; ESCC, esophageal squamous cell carcinoma; RASA1, RAS p21 protein activator 1.

Construction of a tumor-burdened mouse model and in vivo treatment. The Eca-109 $\left(2 \times 10^{6}\right)$ cells were subcutaneously injected in the posterior right gluteal area. When the tumor volume reached $\sim 0.1 \mathrm{~mm}^{3}$, the mice were randomly assigned into 2 groups. One group received an injection with antagomir $\mathrm{NC}(10 \mathrm{nmol}, 50 \mu \mathrm{l})$ and the other antagomir $(10 \mathrm{nmol}, 50 \mu \mathrm{l})$ every 3 days with 5 mice in each group. The tumor volume was measured before and every 3 days after treatment. The volume was calculated with the following formula: $\mathrm{V}=1 / 2$ $\left(\mathrm{a} \times \mathrm{b}^{2}\right)$, in which a was the long diameter and $\mathrm{b}$ was the short diameter of the tumor. Twenty-one days after treatment, the mice were euthanized with $\mathrm{CO}_{2}$ and the tumor tissues were dissected.

Data analysis. The data was calculated and analyzed with Excel and SPSS 13.0 packages (SPSS, Inc., Chicago, IL, USA). Data was expressed as the mean \pm standard deviation (SD) of separate experiments. Statistical analysis was presented by the repeated measure, independent-samples t-test and one-way analysis of variance (ANOVA) followed by Dunnett's post hoc test. $\mathrm{P}<0.05$ was regarded as a significant difference.

\section{Results}

Identification DE miRNAs. A total of $627 \mathrm{DE}$ miRNAs were identified. The top $20 \mathrm{DE}$ miRNAs (top 20 upregulated and 20 downregulated miRNAs) was analyzed with the Morpheus online tool to form a heat map, which was screened by SNR value. The heat map revealed that miR-21 was one of the principal genes and was selected for further research (Fig. 1).

Overexpressed miR-21 enhances the cell colony formation rate and cell healing rate. The cell colonies assay revealed that the cell colony formation rate was significantly decreased in the miR-21 inhibitor group compared with the inhibitor NC group, while the rate was significantly increased in the miR-21 mimic group compared with the mimic NC group $(\mathrm{P}<0.05)$ (Fig. 2). The results of the scratch assay revealed that, the cell healing 
A

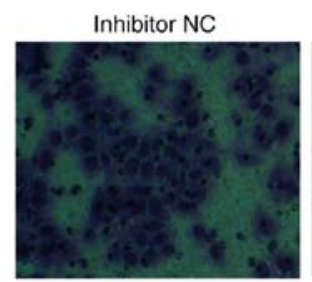

B

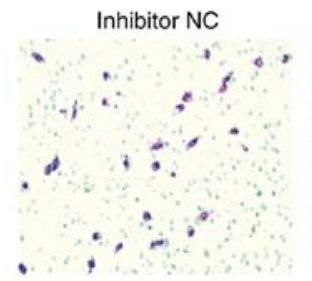

C

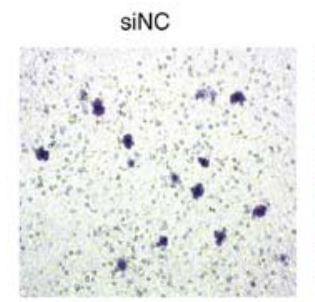

$\mathrm{E}$

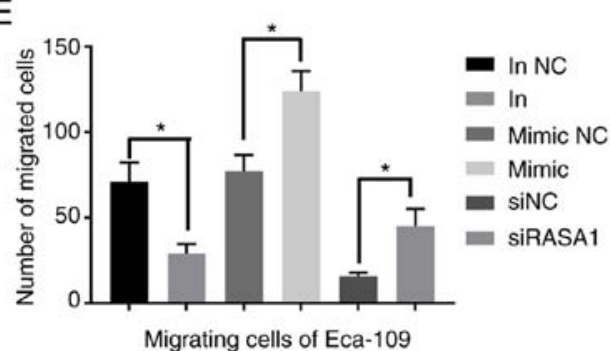

Inhibitor

Inhibitor

SIRASA1
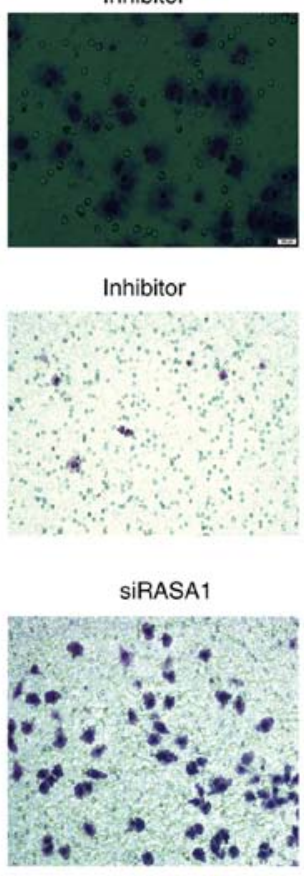

D $\quad$ sinc
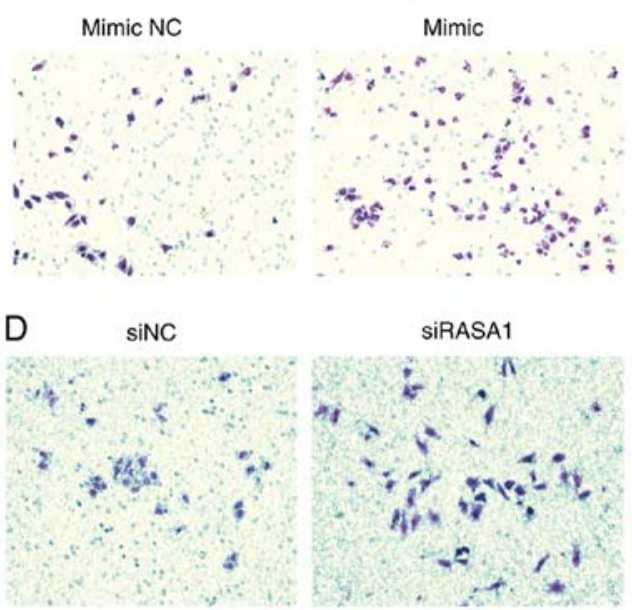

$\mathrm{F}$

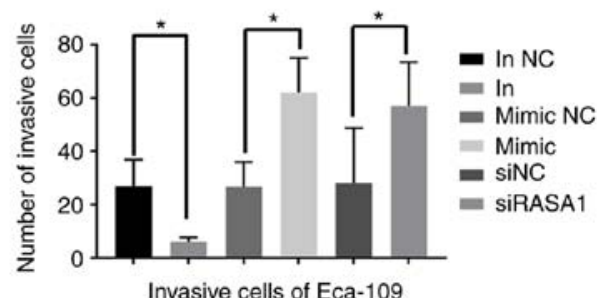

Figure 3. Transwell assay of miR-21 and RASA1 on ESCC cell line Eca-109 in vitro. (A and C) Cells from a migration assay viewed under a microscope (magnification in A was x400, magnification in C was x200). (B and D) Cells from an invasion assay viewed under a microscope (magnification, $\mathrm{x} 200$ ). (E) The number of migrated cells were significantly decreased in the miR-21 inhibitor group compared with the inhibitor NC group, while they were significantly increased in the miR-21 mimic group compared with the mimic NC group, and the siRASA1 group compared with the siNC group. (F) The number of invasive cells was significantly decreased in the miR-21 inhibitor group compared with the inhibitor NC group, while they were significantly increased in the miR-21 mimic group compared with the mimic NC group, and the siRASA1 group compared with the siNC group ( $\left.{ }^{*}<<0.05\right)$. miR-21, microRNA-21; ESCC, esophageal squamous cell carcinoma; RASA1, RAS p21 protein activator 1.

rate was significantly inhibited in the miR-21 inhibitor group compared with the inhibitor NC group after the cells were transfected for $48 \mathrm{~h}$. Conversely, the healing rate was significantly enhanced in the miR-21 mimic group and the siRAS1 group compared with the mimic NC and siNC group, respectively (all $\mathrm{P}<0.05)$ (Fig. 2).

Cell migration and invasion assay. The number of migrated and invasive cells was significantly decreased in the miR-21 inhibitor group compared with the inhibitor NC group (all $\mathrm{P}<0.05)$. Conversely, the cells were significantly increased in the miR-21 mimic group compared with the mimic NC group (all $\mathrm{P}<0.05$ ). Notably, we observed that the number of migrated and invasive Eca-109 cells was also significantly increased in the siRASA1 group compared with the siNC group (all $\mathrm{P}<0.05$ ). Similar results were also obtained with the KYSE510 cells (Figs. 3 and 4).

Construction of a vector for RASA1. The sequence of RASA1 3'UTR which was combined to miR-21 (mature sequence: UAGCUUAUCAGACUGAUGUUGA) was amplified and detected at 1043 bp using electrophoresis. The vector with mutated_or wild-type RASA1 was constructed and used for the luciferase reporter assay (Fig. 5A-C).

Dual-luciferase reporter assay. The result of the dual-luciferase reporter assay revealed that the luciferase activity in the miR-21 group transfected with the RASA1 vector was significantly inhibited compared with the NC and the blank group $(\mathrm{P}<0.05)$ (Fig. 5D), while the activity in the miR-21 group transfected with the RASA1 mutated vector was not significantly altered compared with the $\mathrm{NC}$ and the blank group ( $\mathrm{P}>0.05)$ (Fig. 5E).

$q-P C R$ results. It predicted that RASA1 may be the target gene of miR-21 by miRWalk. Their relative expression by PCR revealed that miR-21 was significantly increased and RASA1 was decreased in the tumor tissues compared to the normal adjacent tissues in patients with ESCC (Fig. 1B). The gene level of RASA1 was significantly increased when the Eca-109 cells were transfected with the miR-21 inhibitor compared with the inhibitor NC $(\mathrm{P}<0.05)$. Conversely, the expression 
A
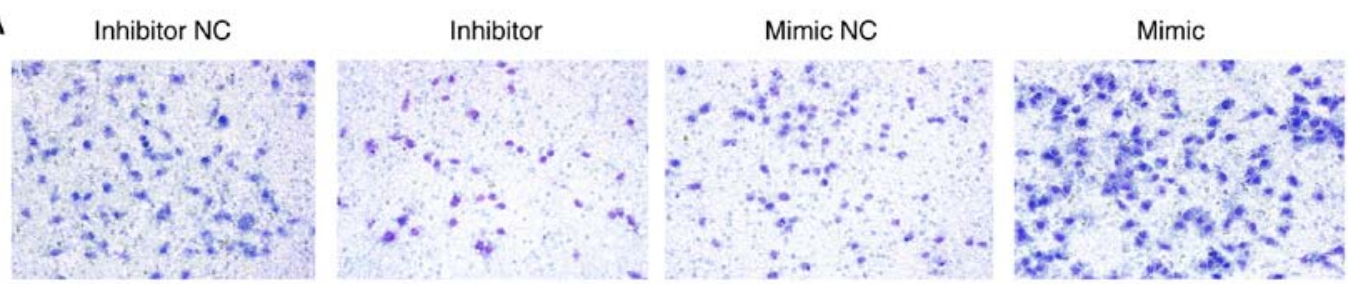

B
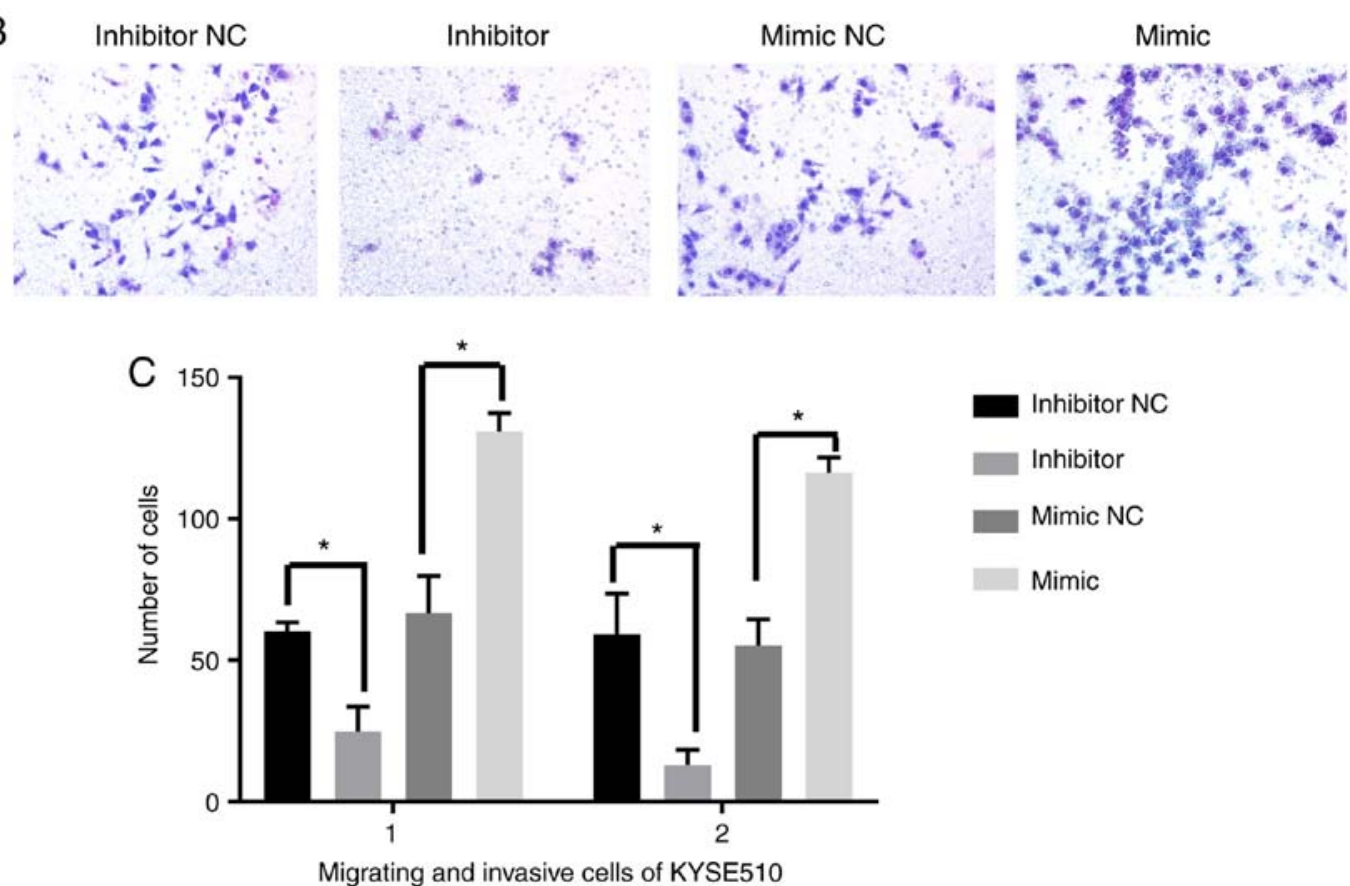

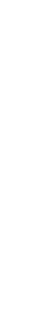


A

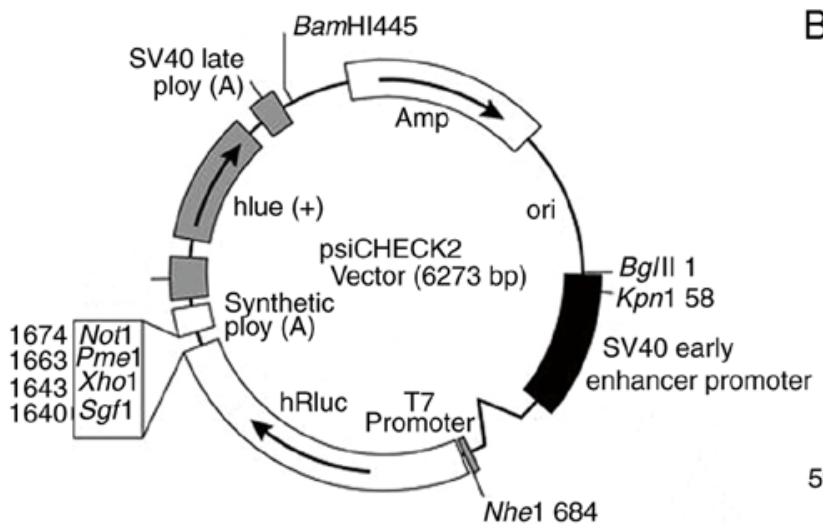

C

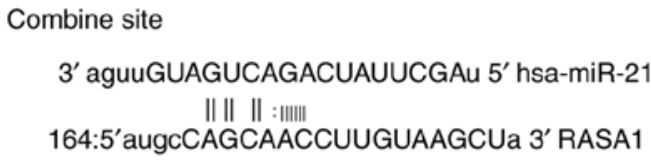

Combine site

$3^{\prime}$ aguuGUAGUCAGACUAUUCGAu $5^{\prime}$ hsa-miR-21 || || || :|||||| 164:5'augcCAGCAACCUUGUAAGCUa 3' RASA1

D

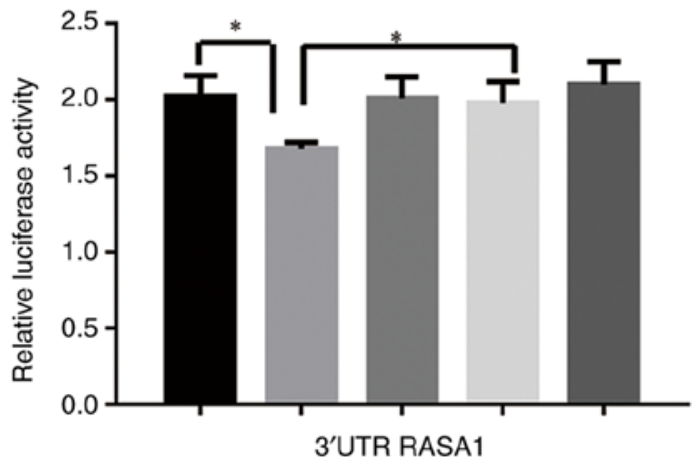

$E$

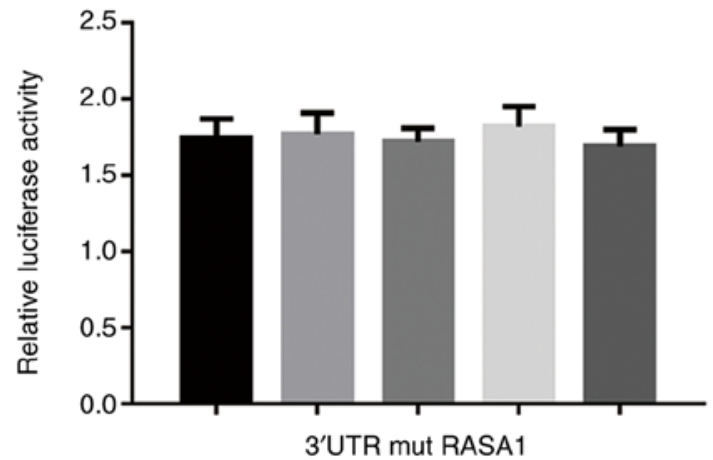

$\mathrm{F}$

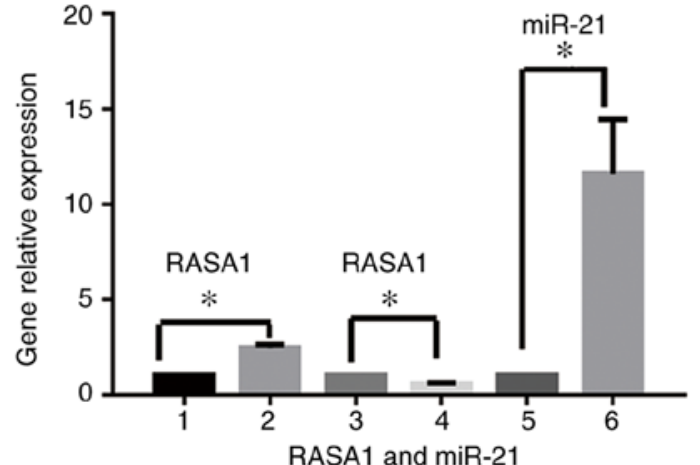

B
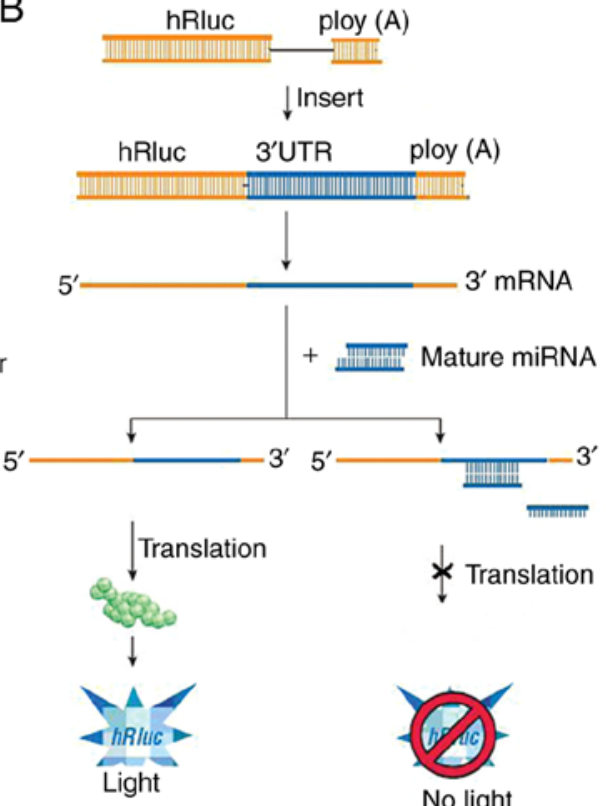

$\ngtr$ Translation

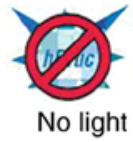

No light

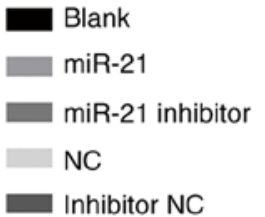

miR-21 inhibitor NC

miR-21 inhibitor

miR-21 mimic NC

miR-21 mimic

siNC

SiRASA1

Figure 5. Interaction between miR-21 and RASA1 is detected by luciferase assay and q-PCR. (A) The construction of the vector. (B) The mechanism of the luciferase assay. (C) The combined site of RASA1 and miR-21. (D) The luciferase activity in the miR-21 group transfected with RASA1 vector was significantly inhibited compared with the NC and blank group. (E) The activity in the miR-21 group transfected with RASA1 mutated vector was not significantly altered compared with the NC and the blank group. (F) The expression of RASA1 was significantly increased in the Eca-109 cell transfected with the miR-21 inhibitor compared with the inhibitor NC, and decreased in the miR-21 mimic group when compared with the mimic NC group. In addition, the expression of miR-21 was significantly increased in the group of cells which were transfected with siRASA1 when compared with the siNC group ("P<0.05). miR-21, microRNA-21; RASA1, RAS p21 protein activator 1; q-PCR, quantitative polymerase chain reaction. 


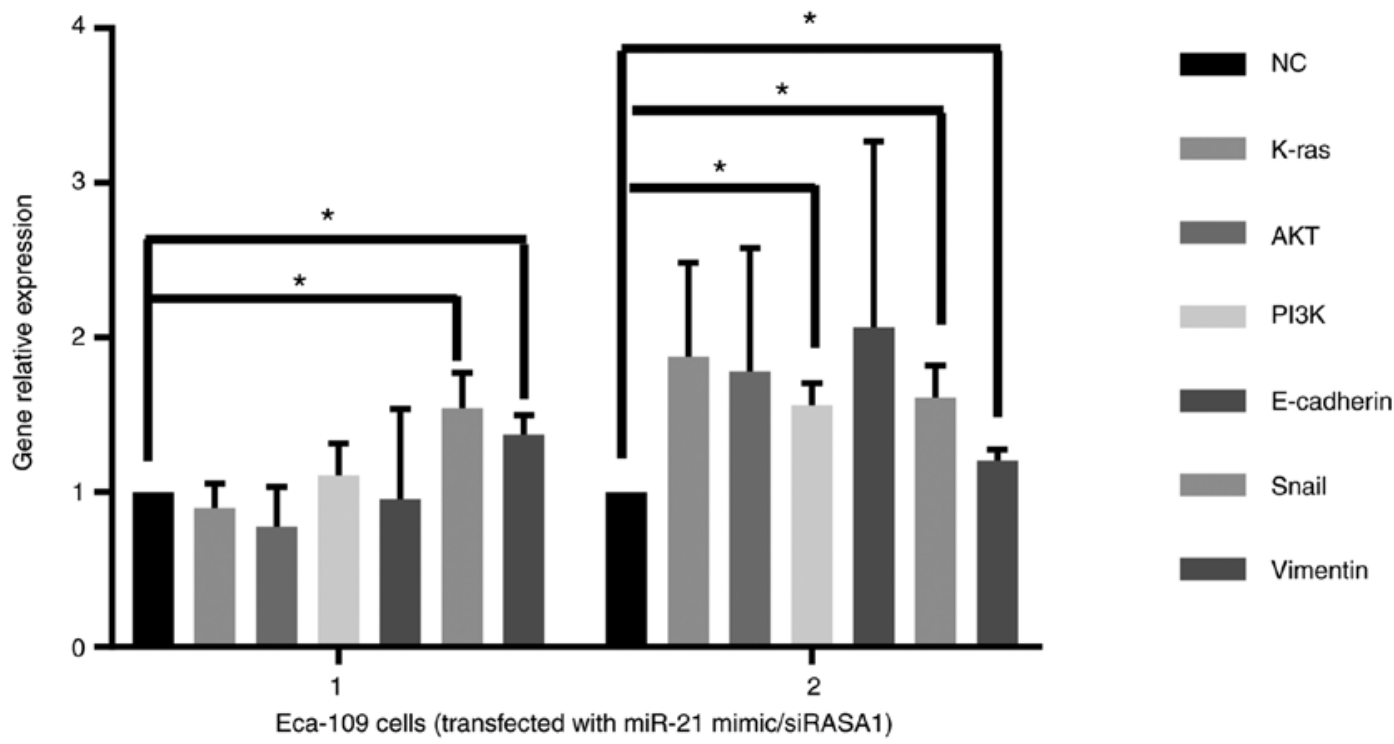

Figure 6. The relative gene expression. Group 1, the relative gene expression of signaling molecules in ESCC cell line Eca-109, with upregulated miR-21 (mimic). Group 2, the relative gene expression of signaling molecules in Eca-109 cells with downregulated of RASA1 (siRASA1). The relative gene expression of Snail and vimentin were significantly increased by upregulated miR-21 or downregulated RASA1 ("P $<0.05)$. miR-21, microRNA-21; ESCC, esophageal squamous cell carcinoma; RASA1, RAS p21 protein activator 1.

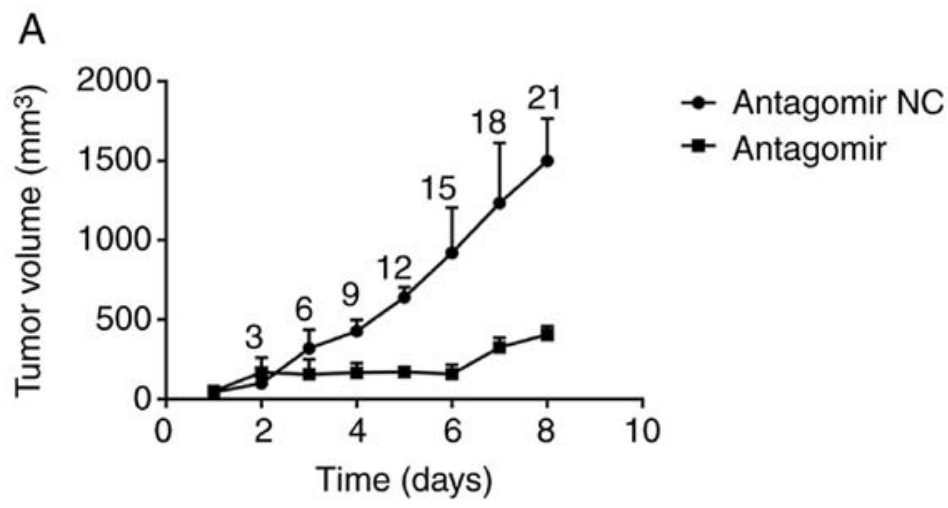

B

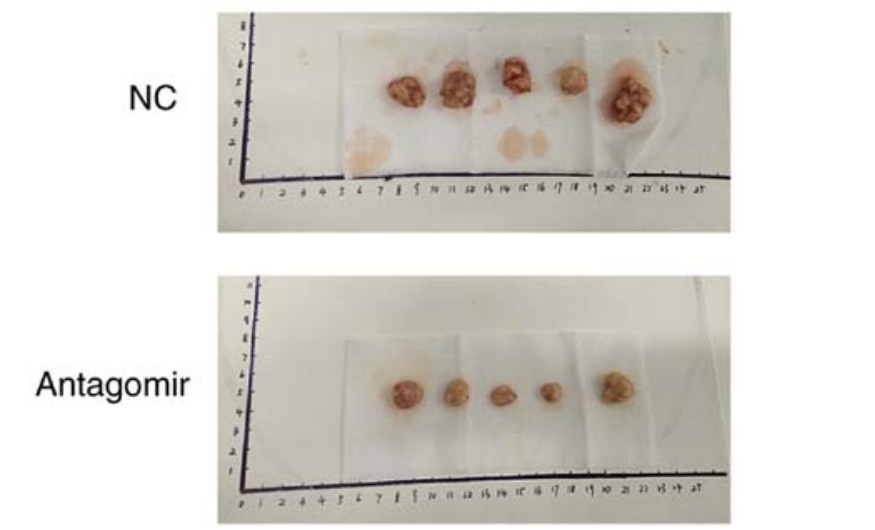

Figure 7. The effect of miR-21 on tumor xenografts. (A) Nude mice bearing tumors were established to evaluate the effects of miR-21 inhibitor (antagomir) on esophageal carcinoma growth in vivo $\left(\mathrm{mm}^{3}\right)$. Application of miR-21 antagomir significantly inhibited the growth of the tumors compared with the NC group, 3 and 6 days after treatment, respectively $(\mathrm{P}<0.05)$. The tumor volume was not significant different before treatment $(\mathrm{P}>0.05)$. (B) The tumor masses excised from the nude mice. miR-21, microRNA-21; ESCC, esophageal squamous cell carcinoma; RASA1, RAS p21 protein activator 1.

one of the 20 top DE miRNAs (upregulated) in patients with ESCC. Our results also revealed that the relative expression of miR-21 was significantly increased in tumor tissues compared to normal adjacent tissues. Furthermore, the results revealed that RASA1 may be a target gene of miR-21 and that miR-21 and its target gene may play an important role in regulating ESCC. Thus, a functional experiment of miR-21 and RASA1 was performed on ESCC cell lines. Notably, we observed that 
overexpressed miR-21 could significantly enhance the healing rate and increase the number of invasive and migrated cells as well as the cell colony formation rate. Conversely, downregulation of the expression of miR-21 could significantly inhibit the healing rate and decrease the number of invasive and migrated cells as well as the cell colony formation rate. Futhermore, downregulated RASA1 (siRASA1), significantly increased the number of invasive and migrated cells. These results indicated that miR-21 and its target gene RASA1 regulated cell invasion, migration and proliferation of ESCC cells.

It has been reported that miRNAs negatively regulate their target gene via binding to the 3'UTR of target mRNAs, which causes mRNA degradation suppressing translation (31). In the present study, we observed that overexpression of miR-21 significantly decreased the expression of RASA1. Conversely, downregulated miR-21 significantly increased the RASA1 gene expression. These results revealed that miR-21 negatively regulated RASA1. In order to further elucidate the relationship between RASA1 and miR-21, we constructed an mRNA vector of RASA1 combined to mature miR-21 and performed a luciferase reporter assay. The assay revealed that RASA1 was combined with miR-21. With the PCR results, the relative gene expression of Snail and vimentin was significantly increased by upregulated miR-21 or downregulated RASA1. These results indicated that miR-21 directly targeted RASA1, and that the regulatory role may be via Snail and vimentin. RASA1 was firstly discovered as a RAS guanosine triphosphate enzyme-activated protein, which inactivated RAS to inhibit the RAS downstream pathway, thereby resulting in the proliferation, migration, invasion and metastasis of cancer cells. Snail is a pivotal gene which is related to EMT. Thus, we hypothesized that miR-21 regulated migration and invasion by directly targeting RASA1 which may be via regulation of Snail. However, the precise mechanism in the downstream pathway requires further research.

In order to investigate the effect of miR-21 in vivo, we set up a nude mouse tumor-bearing model. We observed that application of miR-21 inhibitor significantly inhibited the growth of the tumor compared to the NC group at days 3 and 6 after treatment, respectively. This indicated that anti-miR-21 had an antitumor effect and may be considered as a promising target for ESCC therapy.

In conclusion, miR-21 regulated cell proliferation, migration, invasion and tumor growth of ESCC by directly targeting RASA1, possibly via regulation of Snail. miR-21 and RASA1 may be considered as possible targets for ESCC therapy.

\section{Acknowledgements}

The authors thank Professor Edward I. Wong (Milton International Education Group, Hong Kong) for revising the manuscript.

\section{Funding}

The present study was supported by grants from the Technical New Star of Zhujiang, Panyu District, Guangzhou (2013special-15-6.10), the Science and Technology Program of Panyu (2015-Z03-09) and the Science and Technology Program of Guangzhou (grant no. 201804010012).

\section{Availability of data and materials}

The data used during the study are available from the corresponding author upon reasonable request.

\section{Authors' contributions}

$\mathrm{XCh}$ and SC performed the analysis of the data and wrote the paper. XCa and XZ performed the data analysis of GEO. LW and HL collected the samples and the experimental data. SC, BL, LW, WL and ZW performed the experiments. All authors read and approved the manuscript and agree to be accountable for all aspects of the research in ensuring that the accuracy or integrity of any part of the work are appropriately investigated and resolved.

\section{Ethics approval and consent to participate}

This study was approved by the Ethics Committee of Panyu Central Hospital (Guangzhou, China). All patients provided informed consent to participate in this study. The animal experiments were approved by the Ethics Committee of Panyu Central Hospital (no. K20170002).

\section{Patient consent for publication}

Not applicable.

\section{Competing interests}

The authors declare that they have no competing interests.

\section{References}

1. Siegel RL, Miller KD and Jemal A: Cancer statistics, 2017. CA Cancer J Clin 67: 7-30, 2017.

2. Tran GD, Sun XD, Abnet CC, Fan JH, Dawsey SM, Dong ZW, Mark SD, Qiao YL and Taylor PR: Prospective study of risk factors for esophageal and gastric cancers in the Linxian general population trial cohort in China. Int J Cancer 113: 456-463, 2005

3. Ando N, Kato H, Igaki H, Shinoda M, Ozawa S, Shimizu H, Nakamura T, Yabusaki H, Aoyama N, Kurita A, et al: A randomized trial comparing postoperative adjuvant chemotherapy with cisplatin and 5-fluorouracil versus preoperative chemotherapy for localized advanced squamous cell carcinoma of the thoracic esophagus (JCOG9907). Ann Surg Oncol 19: 68-74, 2012.

4. Yu EH, Tu HF, Wu CH, Yang CC and Chang KW: MicroRNA-21 promotes perineural invasion and impacts survival in patients with oral carcinoma. J Chin Med Assoc 80: 383-388, 2017.

5. Teoh SL and Das S: The role of microRNAs in diagnosis, prognosis, metastasis and resistant cases in breast cancer. Curr Pharm Des 23: 1845-1859, 2017.

6. Tseng HH, Tseng YK, You JJ, Kang BH, Wang TH, Yang CM, Chen HC, Liou HH, Liu PF, Ger LP, et al: Next-generation sequencing for microRNA profiling: MicroRNA-21-3p promotes oral cancer metastasis. Anticancer Res 37: 1059-1066, 2017.

7. Cao J, Liu J, Xu R, Zhu X, Liu L and Zhao X: MicroRNA-21 stimulates epithelial-to-mesenchymal transition and tumorigenesis in clear cell renal cells. Mol Med Rep 13: 75-82, 2016.

8. Li P, Mao WM, Zheng ZG, Dong ZM and Ling ZQ: Down-regulation of PTEN expression modulated by dysregulated miR-21 contributes to the progression of esophageal cancer. Dig Dis Sci 58: 3483-3493, 2013.

9. Shen KH, Hung JH, Chang CW, Weng YT, Wu MJ and Chen PS: Solasodine inhibits invasion of human lung cancer cell through downregulation of miR-21 and MMPs expression. Chem Biol Interact 268: 129-135, 2017. 
10. Chen Z, Yu T, Cabay RJ, Jin Y, Mahjabeen I, Luan X, Huang L, Dai $Y$ and Zhou X: miR-486-3p, miR-139-5p, and miR-21 as biomarkers for the detection of oral tongue squamous cell carcinoma. Biomark Cancer 9: 1-8, 2017.

11. Mei LL, Qiu YT,Zhang B and Shi ZZ: MicroRNAs in esophageal squamous cell carcinoma: Potential biomarkers and therapeutic targets. Cancer Biomark 19: 1-9, 2017.

12. He Y, Jin J, Wang L, Hu Y, Liang D, Yang H, Liu Y and Shan B: Evaluation of miR-21 and miR-375 as prognostic biomarkers in oesophageal cancer in high-risk areas in China. Clin Exp Metastasis 34: 73-84, 2017.

13. Han JG, Jiang YD, Zhang CH, Yang YM, Pang D, Song YN and Zhang GQ: A novel panel of serum miR-21/miR-155/miR-365 as a potential diagnostic biomarker for breast cancer. Ann Surg Treat Res 92: 55-66, 2017.

14. Markou A, Zavridou M and Lianidou ES: miRNA-21 as a novel therapeutic target in lung cancer. Lung Cancer 7: 19-27, 2016.

15. Dong J, Zhang Z, Gu T, Xu SF, Dong LX, Li X, Fu BH and Fu ZZ: The role of microRNA-21 in predicting brain metastases from non-small cell lung cancer. Onco Targets Ther 10: 185-194, 2017.

16. Ren J, Kuang TH, Chen J, Yang JW and Liu YX: The diagnostic and prognostic values of microRNA-21 in patients with gastric cancer: A meta-analysis. Eur Rev Med Pharmacol Sci 21: 120-130, 2017

17. Arantes LM, Laus AC, Melendez ME, de Carvalho AC, SorrocheBP,De MarchiPR,Evangelista AF,Scapulatempo-NetoC, de Souza Viana L and Carvalho AL: MiR-21 as prognostic biomarker in head and neck squamous cell carcinoma patients undergoing an organ preservation protocol. Oncotarget 8 : 9911-9921, 2017.

18. Yang Y, Guo JX and Shao ZQ: miR-21 targets and inhibits tumor suppressor gene PTEN to promote prostate cancer cell proliferation and invasion: An experimental study. Asian Pac J Trop Med 10: 87-91, 2017.

19. Huo W, Zhao G, Yin J, Ouyang X, Wang Y, Yang C, Wang B, Dong P, Wang Z, Watari H, et al: Lentiviral CRISPR/Cas9 vector mediated miR-21 gene editing inhibits the epithelial to mesenchymal transition in ovarian cancer cells. J Cancer 8: 57-64, 2017.

20. Liu CH, Huang Q, Jin ZY, Zhu CL, Liu Zand Wang C: miR-21 and KLF4 jointly augment epithelial-mesenchymal transition via the Akt/ERK1/2 pathway. Int J Oncol 50: 1109-1115, 2017.

21. Li C, Song L, Zhang Z, Bai XX, Cui MF and Ma LJ: MicroRNA-21 promotes TGF- $\beta 1$-induced epithelial-mesenchymal transition in gastric cancer through up-regulating PTEN expression. Oncotarget 7: 66989-67003, 2016.

22. Yan L, Cao R, Liu Y, Wang L, Pan B, Lv X, Jiao H, Zhuang Q, Sun X and Xiao R: MiR-21-5p links epithelial-mesenchymal transition phenotype with stem-like cell signatures via AKT signaling in keloid keratinocytes. Sci Rep 6: 28281, 2016.

23. Sun SS, Zhou X, Huang YY, Kong LP, Mei M, Guo WY,Zhao MH, Ren Y, Shen Q and Zhang L: Targeting STAT3/miR-21 axis inhibits epithelial-mesenchymal transition via regulating CDK5 in head and neck squamous cell carcinoma. Mol Cancer 14: 213, 2015.

24. Wu ZH, Tao ZH, Zhang J, Li T, Ni C, Xie J, Zhang JF and Hu XC: MiRNA-21 induces epithelial to mesenchymal transition and gemcitabine resistance via the PTEN/AKT pathway in breast cancer. Tumour Biol 37: 7245-7254, 2016.
25. Liu Z, Jin ZY, Liu CH, Xie F, Lin XS and Huang Q: MicroRNA-21 regulates biological behavior by inducing EMT in human cholangiocarcinoma. Int J Clin Exp Pathol 8: 4684-4694, 2015.

26. Bornachea O, Santos M, Martínez-Cruz AB, García-Escudero R, Dueñas M,Costa C, Segrelles C,Lorz C, Buitrago A, Saiz-Ladera C, et al: EMT and induction of miR-21 mediate metastasis development in Trp53-deficient tumours. Sci Rep 2: 434, 2012.

27. Han M, Wang Y, Liu M, Bi X, Bao J, Zeng N, Zhu Z, Mo Z, Wu C and Chen X: MiR-21 regulates epithelial-mesenchymal transition phenotype and hypoxia-inducible factor-1alpha expression in third-sphere forming breast cancer stem cell-like cells. Cancer Sci 103: 1058-1064, 2012

28. Mathé EA, Nguyen GH, Bowman ED, Zhao Y, Budhu A, Schetter AJ, Braun R, Reimers M, Kumamoto K, Hughes D, et al: MicroRNA expression in squamous cell carcinoma and adenocarcinoma of the esophagus: Associations with survival. Clin Cancer Res 15: 6192-6200, 2009.

29. Livak KJ and Schmittgen TD: Analysis of relative gene expression data using real-time quantitative PCR and the $2^{-\Delta \Delta C_{\mathrm{T}}}$ method. Methods 25: 402-408, 2001

30. Winther M, Alsner J, Tramm T, Baeksgaard L, Holtved E and Nordsmark M: Evaluation of miR-21 and miR-375 as prognostic biomarkers in esophageal cancer. Acta Oncol 54: 1582-1591, 2015.

31. Lv H, He Z, Wang H, Du T and Pang Z: Differential expression of miR-21 and miR-75 in esophageal carcinoma patients and its clinical implication. Am J Transl Res 8: 3288-3298, 2016.

32. Sheikh AM, Small HY, Currie G and Delles C: Systematic review of Micro-RNA expression in pre-eclampsia identifies a number of common pathways associated with the disease. PLoS One 11: e0160808, 2016.

33. Yan LX, Liu YH, Xiang JW, Wu QN, Xu LB, Luo XL, Zhu XL, Liu C, Xu FP, Luo DL, et al: PIK3R1 targeting by miR-21 suppresses tumor cell migration and invasion by reducing PI3K/AKT signaling and reversing EMT, and predicts clinical outcome of breast cancer. Int J Oncol 48: 471-484, 2016.

34. Cufi S, Bonavia R, Vazquez-Martin A, Oliveras-Ferraros C, Corominas-Faja $\mathrm{B}$, Cuyàs $\mathrm{E}$, Martin-Castillo $\mathrm{B}$, BarrajónCatalán E, Visa J, Segura-Carretero A, et al: Silibinin suppresses EMT-driven erlotinib resistance by reversing the high $m i R-21 /$ low $m i R-200 c$ signature in vivo. Sci Rep 3: 2459, 2013.

35. Han M, Liu M, Wang Y, Chen X, Xu J, Sun Y, Zhao L, Qu H, Fan Y and $\mathrm{Wu} \mathrm{C}$ : Antagonism of miR-21 reverses epithelial-mesenchymal transition and cancer stem cell phenotype through AKT/ERK1/2 inactivation by targeting PTEN. PLoS One 7: e39520, 2012.

This work is licensed under a Creative Commons Attribution-NonCommercial-NoDerivatives 4.0 International (CC BY-NC-ND 4.0) License. 